\title{
A non Standard Model Higgs at the LHC as a sign of naturalness
}

\author{
Asimina Arvanitaki $^{a}$ and Giovanni Villadoro ${ }^{b}$ \\ a Stanford Institute for Theoretical Physics, Stanford University, \\ 382 Via Pueblo Mall, Stanford, CA 94305 U.S.A. \\ ${ }^{b}$ SLAC, Stanford University, \\ 2575 Sand Hill Rd., Menlo Park, CA 94025 U.S.A. \\ E-mail: aarvan@stanford.edu, giovanni.villadoro@cern.ch
}

ABSTRACT: Light states associated with the hierarchy problem affect the Higgs LHC production and decays. We illustrate this within the MSSM and two simple extensions applying the latest bounds from LHC Higgs searches. Large deviations in the Higgs properties are expected in a natural SUSY spectrum. The discovery of a non-Standard-Model Higgs may signal the presence of light stops accessible at the LHC. Conversely, the more the Higgs is Standard-Model-like, the more tuned the theory becomes. Taking the ratio of different Higgs decay channels at the LHC cancels the leading QCD uncertainties and potentially improves the accuracy in Higgs coupling measurements to the percent level. This may lead to the possibility of doing precision Higgs physics at the LHC. Finally, we entertain the possibility that the ATLAS excess around $125 \mathrm{GeV}$ persists with a Higgs production crosssection that is enhanced compared to the SM. This increase can only be accommodated in extensions of the MSSM and it may suggest that stops lie below $400 \mathrm{GeV}$, likely within reach of next year's LHC run.

Keywords: Higgs Physics, Beyond Standard Model, Supersymmetric Standard Model

ARXIV EPRINT: 1112.4835 


\section{Contents}

1 Introduction 1

2 The Higgs in supersymmetric models 2

2.1 Collider bounds 5

2.2 Bounds from $b \rightarrow s \gamma \quad 6$

3 The SUSY Higgs at the LHC $\quad 6$

3.1 The Higgs in the MSSM 8

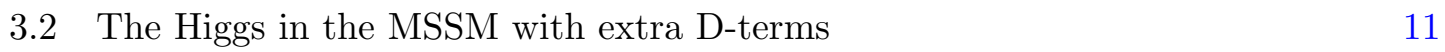

3.3 The Higgs in the NMSSM 13

$\begin{array}{ll}3.4 & \text { Large mixing between Higgs states } \\ & 3.4 .15\end{array}$

$\begin{array}{lll}3.4 .1 & \text { Mixing in the MSSM } & 15\end{array}$

$\begin{array}{ll}\text { 3.4.2 Mixing in the NMSSM } & 17\end{array}$

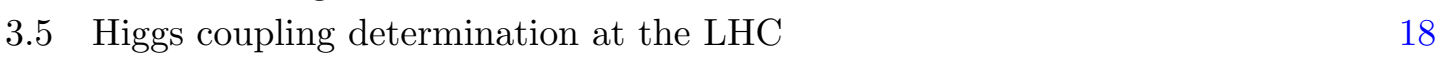

$\begin{array}{lll}3.6 & \text { The possibility of a } 125 \mathrm{GeV} \text { Higgs } & 18\end{array}$

$\begin{array}{llr}4 \text { Conclusions } & 19\end{array}$

\section{Introduction}

The Higgs boson is the last missing ingredient of the Standard Model (SM) and it has been the focus of collider searches in the past 30 years. It explains electroweak (EW) symmetry breaking and the particle masses but at the price of a huge fine-tuning needed to keep the scale of weak interactions far below the Planck scale. Any solution to this hierarchy problem requires new degrees of freedom at the EW scale to cancel the quadratic divergences of the Higgs:

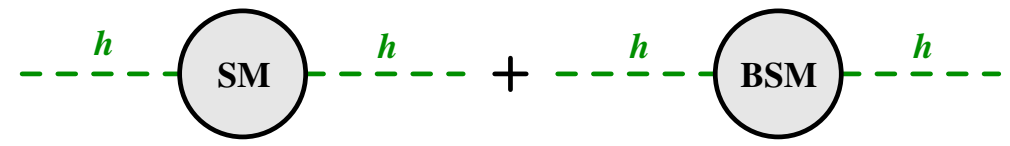

These degrees of freedom also affect the main Higgs production mechanism at the Large Hadron Collider (LHC), gluon-gluon fusion:

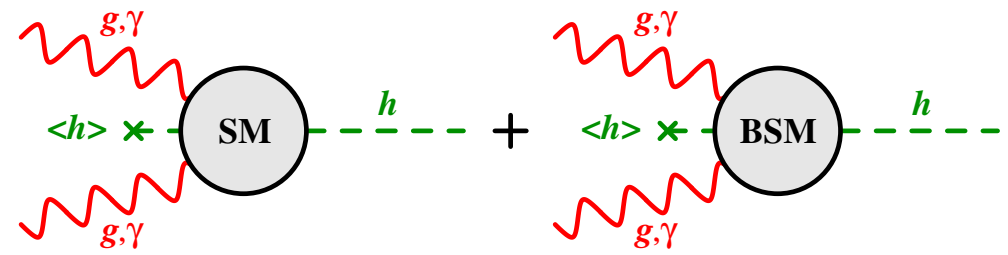


The same is true for one of the most important Higgs decay channels, $h \rightarrow \gamma \gamma$. Therefore we expect a natural Higgs at the LHC to behave differently from the SM Higgs. Vice-versa the more the Higgs is SM-like the more tuned the theory will be. This fact highlights the importance of accurately measuring the Higgs couplings to SM particles.

In this paper we study the interplay between naturalness and Higgs properties at the LHC in the framework of supersymmetry (SUSY) [1] . Supersymmetry is still the leading candidate for physics beyond the SM, it predicts gauge coupling unification, and provides a viable dark matter candidate. How natural is SUSY, though, given the absence of any significant deviation from the SM? Within the minimal implementation of SUSY (MSSM), the LEP bounds on the Higgs mass increase the fine-tuning to an uncomfortable level, but this problem is solved in simple extensions of the MSSM. Still, current LHC bounds on squark masses naively push the scale of SUSY around $1 \mathrm{TeV}[2,3]$ and reintroduce the fine-tuning problem. These bounds, however, apply for universal squark masses, while naturalness in SUSY requires only the top squarks to be light [4]. Direct searches for third family sparticles allow for stops as light as the top and SUSY can be natural [5-7]. In this case light stops can significantly alter LHC Higgs production and decay.

Besides the stop sector, the tree level Higgs couplings to SM particles also depend on the mixing of the light Higgs with the other Higgses in SUSY. The Higgs behavior at the LHC and the tuning can be adequately described by just three parameters: the lightest stop mass, the stop mixing and the Higgs mixing angle. We study how the Higgs production and decay increase or decrease with respect to the SM as a function of these three parameters in the MSSM and two simple extensions. We also discuss how taking the ratio of different decay channels improves the accuracy for Higgs coupling measurements at the LHC. Finally, we comment on the recent ATLAS excess at $\sim 125 \mathrm{GeV}$ and, if confirmed, what this would imply for the SUSY spectrum.

\section{The Higgs in supersymmetric models}

The light Higgs mass in the MSSM is naturally constrained to lie below the $Z$ mass at tree level. The LEP bounds [8] put tremendous strain on SUSY where large radiative corrections are required to raise the Higgs mass. This also increases the amount of tuning that is needed to account for the measured $G_{F}$. The amount of tuning can be quantified by the relation [9]:

$$
F T(\xi)=\left[\sum_{i}\left(\frac{\partial \log v^{2}}{\partial \log \xi^{i}}\right)^{2}\right]^{-1 / 2},
$$

where $\xi_{i}$ includes all the parameters that contribute to $v$. In the MSSM, the $Z$ mass is, in the large $\tan \beta$ limit:

$$
m_{Z}^{2} \approx-2\left(m_{H_{u}}^{2}+\mu^{2}\right)+\frac{2}{\tan ^{2} \beta}\left(m_{H_{d}}^{2}-m_{H_{u}}^{2}\right) .
$$

As can be seen from this formula, the main contributors to the tuning are the $\mu$-term and $m_{H_{u}} \cdot m_{H_{u}}$ also receive large radiative corrections,

$$
\delta m_{H_{u}}^{2} \approx \frac{3 y_{t}^{2}}{16 \pi^{2}}\left(m_{\tilde{t}_{1}}^{2}+m_{\tilde{t}_{2}}^{2}+A_{t}^{2}\right) \log \left(\frac{2 \Lambda^{2}}{m_{\tilde{t}_{1}}^{2}+m_{\tilde{t}_{2}}^{2}}\right),
$$


from the stop masses, $m_{\tilde{t}_{1,2}}$, and mixing, $A_{t}$, which are also the parameters responsible for raising the Higgs mass. As a result, naturalness (say $F T \gtrsim 10 \%$ ) pushes Higgsinos and stops to be light:

$$
\mu \lesssim 250 \mathrm{GeV} \text {, and } m_{\tilde{t}} \lesssim 350 \mathrm{GeV}
$$

It is worth noting that $m_{H_{d}}$ does not affect the tuning significantly at the large $\tan \beta$ limit, so the rest of the Higgs sector in the MSSM can be above $1 \mathrm{TeV}$ without loss of naturalness.

The LHC production of the Higgs is primarily modified by the same particles that contribute the most to the radiative corrections of its mass and to the tuning: the stops. Even though current LHC searches exclude the possibility of universal squark spectra up to almost $1 \mathrm{TeV}(\sim 500 \mathrm{GeV}$ with a heavy neutralino), stops can be perhaps as light as the top in models with non-universal squark masses [4] or when SUSY is hidden, for example see [10]. As a result, stops can significantly influence the Higgs coupling to gluons.

In the supersymmetric limit, light stops increase the LHC Higgs production crosssection [11-15], since the latter is related to the $\beta$-function of $\alpha_{s}$ to which scalar fields contribute with the same sign as fermions $[16,17]$. This behavior persists after SUSY breaking in the absence of stop mixing, while a large mixing between the stop eigenstates interferes destructively with the top loop and the gluon fusion process can be suppressed substantially. Neglecting bottom/sbottom and D-term contributions, the approximate formula for the $g g \rightarrow h$ production cross-section of a light Higgs $\left(m_{H} \lesssim m_{t}\right)$ relative to the Standard Model is

$$
\frac{\sigma(g g \rightarrow h)}{\sigma_{S M}(g g \rightarrow h)} \approx\left[1+\frac{1}{4}\left(\frac{m_{t}^{2}}{m_{\tilde{t}_{1}}^{2}}+\frac{m_{t}^{2}}{m_{\tilde{t}_{2}}^{2}}-\frac{m_{t}^{2}\left(A_{t}+\mu / \tan \beta\right)\left(A_{t}-\mu \tan \alpha\right)}{m_{\tilde{t}_{1}}^{2} m_{\tilde{t}_{2}}^{2}}\right)\right]^{2},
$$

where $m_{\tilde{t}_{i}}$ are the stop mass eigenvalues and $\alpha$ is the mixing angle between the up and down type Higgses. This effect can be significant even for stops as heavy as $400 \mathrm{GeV}$ ( $\sim 20 \%$ in the absence of mixing). Notice that the mixing term is always negative since $\left(A_{t}-\mu \tan \alpha\right) \simeq\left(A_{t}+\mu / \tan \beta\right)$, which is a good approximation except in the extreme case of large mixing between the light and the heavy Higgs.

For Higgs masses below $\sim 135 \mathrm{GeV}$, the branching ratio (BR) to photons is also important. Similarly to gluon fusion, the Higgs coupling to two photons is again related to the electroweak $\beta$-functions. In this case, the dominant contribution comes from the $W$ boson while the top gives a smaller contribution with opposite sign. With large $A$-terms, the stop contributions, being opposite to the top, increase the Higgs BR to photons slightly, but this enhancement is smaller than the suppression from the production cross section due to the minor role of the top in the diphoton channel. Chargino states also contribute to $h \rightarrow \gamma \gamma$, but once they become heavier than roughly $100 \mathrm{GeV}$ their effects are subleading.

The other important deviation of the SUSY Higgs' behavior from the SM comes from its natural embedding in two Higgs doublets. The role of the SM Higgs is shared by two Higgs states, $H_{u}$ and $H_{d}$, and the mixing angle $\alpha$ between $H_{u}$ and $H_{d}$ now affects all the tree level couplings of the light scalar Higgs state. The parameters that mostly influence the amount of mixing is the pseudoscalar Higgs mass $m_{A}$ and $\tan \beta$. In the limit of large $\tan \beta$ the shift of the top, bottom and vector boson couplings of the light Higgs compared 
to their SM values are

$$
\begin{aligned}
\frac{\delta y_{t}}{y_{t}} & =\frac{\cos \alpha}{\sin \beta}-1 \approx-\frac{1}{\tan ^{2} \beta} \frac{2 \frac{m_{Z}^{2}}{m_{A}^{2}}}{\left(1-\frac{m_{Z}^{2}}{m_{A}^{2}}\right)^{2}}, \\
\frac{\delta y_{b}}{y_{b}} & =-\frac{\sin \alpha}{\cos \beta}-1 \approx \frac{2 \frac{m_{Z}^{2}}{m_{A}^{2}}}{1-\frac{m_{Z}^{2}}{m_{A}^{2}}}, \\
\frac{\delta g_{V V}}{g_{V V}} & =\sin (\beta-\alpha)-1 \approx-\frac{1}{\tan ^{2} \beta} \frac{2 \frac{m_{Z}^{4}}{m_{A}^{4}}}{\left(1-\frac{m_{Z}^{2}}{m_{A}^{2}}\right)^{2}},
\end{aligned}
$$

respectively. This expansion shows that mixing becomes important for a light Higgs between 115 and $\sim 150 \mathrm{GeV}$, when the BR to $b \bar{b}$ is dominant. Mixing in this case can suppress the Higgs cross-section in a given channel compared to the SM value significantly (more than $20 \%$ ) when $m_{A}$ is lighter than roughly $400 \mathrm{GeV}$. A special region in two Higgs doublet models is the region of maximal mixing, where the role of the SM is almost equally shared between the light and the heavy Higgs, which are now close in mass. The effects of this region will be examined separately in section 3.4.

From the above discussion, it is obvious that the properties of the SUSY Higgs can be adequately described by three parameters: the lightest stop mass $m_{\tilde{t}_{2}}$, the SUSY breaking $A$-term in the stop sector $A_{t}$, and the pseudoscalar Higgs mass $m_{A} \cdot{ }^{1}$ Another parameter that affects the LHC search strategy for the Higgs is its mass. When the Higgs is between 115 and $\sim 135 \mathrm{GeV}$, the most sensitive channel at the LHC is the Higgs decay to two photons. Above those masses, Higgs decay to $W W^{(*)}$ becomes most important and completely dominates the Higgs BR above $2 m_{W} \approx 160 \mathrm{GeV}$. At the same time, the Higgs decay to $Z Z^{(*)}$ increases the LHC sensitivity and becomes the main search channel for Higgs heavier than roughly $200 \mathrm{GeV}$. In what follows we will consider the latest results in Higgs searches [19-25], which are most sensitive in $h \rightarrow \gamma \gamma$ when the Higgs is lighter than $131.5 \mathrm{GeV}$, in $h \rightarrow W W^{(*)}$ for $131.5 \mathrm{GeV}<m_{h}<2 m_{W}$, and the combined $Z Z^{(*)}$ and $W W$ above the WW threshold.

It is well known that, after LEP and Tevatron bounds on the Higgs mass and the sparticle spectrum, within the MSSM the fine-tuning of the parameters is never better than few percent (this is achieved in the golden region of large $A$-terms [18]). The situation improves somewhat in the presence of extra tree-level contributions to the Higgs mass, which relax the tuning of the weak scale. For this reason, in the rest of the paper, we will consider, besides the case of an MSSM Higgs, two other cases: 1) MSSM with extra D-terms (DMSSM), where an extra quartic term for the Higgs is generated by an additional gauge sector that is broken above the electroweak scale [26], and 2) NMSSM [27]( $\lambda S U S Y[28,29])$, where the extra contribution to the Higgs mass comes from an extra singlet (with possibly large couplings).

\footnotetext{
${ }^{1}$ We will not vary the sbottom sector contributions as their effects are only important at extremely large values of $\tan \beta$ and maximal Higgs mixing.
} 


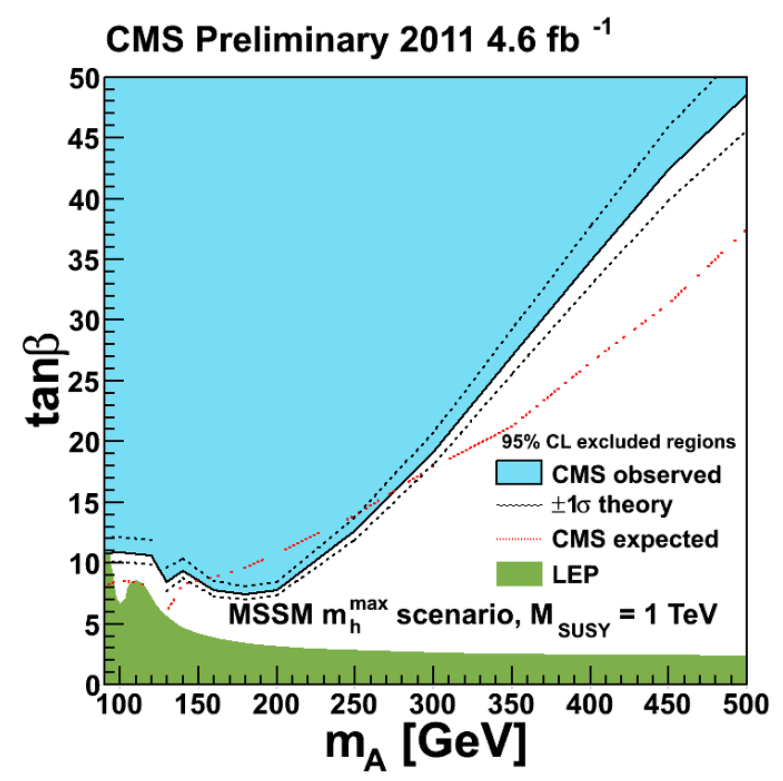

Figure 1. CMS bounds to $m_{A}$ as a function of $\tan \beta$ from [30].

\subsection{Collider bounds}

So far collider searches have focused mainly on universal sparticle spectra with LHC placing bounds that range from 800 to $1000 \mathrm{GeV}$ for squarks masses [2,3]. It is worth pointing out that these bounds are relaxed to roughly $500 \mathrm{GeV}$ when the LSP mass is $200 \mathrm{GeV}$ lighter than the squarks [3]. Still, there are very few searches that focus on the possibility of only a light 3rd family of sparticles. Given the reduced production cross section in this case the bounds are weaker and split family spectra allow for less tuning in SUSY.

At the LHC, 3rd sparticle family searches primarily rely on scalar production from gluino decays. Since the gluino contribution to the Higgs mass enters at two loops, they can be heavier than $\mathrm{TeV}$ and above the present LHC reach. A reanalysis of LHC results [7] suggests that bounds lie around $200-300 \mathrm{GeV}$ depending on whether one or two stops are light but may disappear if the LSP is a pure bino, see also $[5,6]$. The bounds on the stops can also be relaxed in various scenarios where SUSY is hidden. In our analysis we will thus allow $m_{\tilde{t}_{2}}$ to vary from the top mass to roughly $800 \mathrm{GeV}$ as suggested by naturalness.

For the SUSY Higgs sector the biggest constraint comes from the LEP and the LHC searches in the $b \bar{b}$ and $\tau^{+} \tau^{-}$channel respectively [30]. In most of the SUSY parameter space, where there is no large mixing between the light and the heavy Higgs, the light scalar Higgs is constrained to be above $\sim 114 \mathrm{GeV}$. This is the main source of fine-tuning in the MSSM as it naively pushes stop masses above $1 \mathrm{TeV}$. LEP and LHC bounds on the light Higgs also place a bound on $m_{A}$ of roughly $100 \mathrm{GeV}$ (see figure 1).

Charged Higgs searches are not as constraining with LEP bounds on the charged Higgs around $\sim 75 \mathrm{GeV}$ and searches for $t \rightarrow H^{+} b$ at hadron colliders not very sensitive yet [8]. 


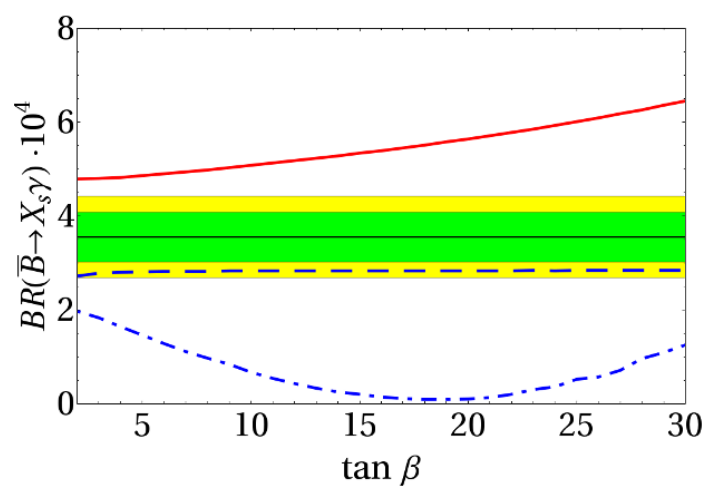

Figure 2. $B R\left(\bar{B} \rightarrow X_{s} \gamma\right)$ vs $\tan \beta$ plot. The green-yellow band correspond to the $1 \sigma-2 \sigma$ experimental bound. All the SUSY particles have been decoupled except 1) $m_{A}=250 \mathrm{GeV}$ for the red line, 2) $\mu=m_{\tilde{t}_{L}}=m_{\tilde{t}_{R}}=200 \mathrm{GeV}, A_{t}=0$ for the dashed blue line, 3) $\mu=m_{\tilde{t}_{L}}=m_{\tilde{t}_{R}}=A_{t}=200 \mathrm{GeV}$ for the dot-dashed blue line.

\subsection{Bounds from $b \rightarrow s \gamma$}

The strongest constraints on a light stop-Higgs sector come from indirect bounds from rare $B$ decays, in particular for the moderate values of $\tan \beta$ considered in this work the constraints are dominated by $b \rightarrow s \gamma$. The main contributions come from the charged Higgs-top and the chargino-stop loops. The charged Higgs increases the amplitude of $b \rightarrow s \gamma$ and varies mildly with $\tan \beta$ unless $\tan \beta \lesssim 1$. When all other SUSY particles are decoupled, the charged Higgs is constrained to be heavier than $\sim 350 \mathrm{GeV}$.

This bound changes drastically when charginos and stops are light [31]. Chargino contributions interfere destructively or constructively with the SM depending on the relative sign of the $\mu$ and $A$-terms. They significantly affect $b \rightarrow s \gamma$ at large $\tan \beta$. Their effect is minimized when the rest of the squarks are also light and the $A$-term are small due to the GIM mechanism. A natural spectrum requires light Higgsinos and stops, and large cancellations from a light charged Higgs or from $A$-terms are necessary to satisfy the current experimental bounds. To illustrate the impact of the different contributions in figure 2 we plot $B R\left(\bar{B} \rightarrow X_{s} \gamma\right)$ with respect to $\tan \beta$ for the three cases where 1) only the charged Higgs contribution is important, 2) only the chargino-stop loop without $A$-terms is important 3 ) only the chargino-stop loop with $A$-terms is important.

\section{The SUSY Higgs at the LHC}

For our analysis and plots we use FeynHiggs [32-35] for the Higgs mass, both for the MSSM and the DMSSM, while we use the one-loop formula from [36] for the NMSSM. Note that there is at least a $2 \mathrm{GeV}$ uncertainty in the Higgs mass, which is larger in the NMSSM case. Depending on the Higgs mass, we consider $\sigma \times B R$ for different channels normalized to the SM values as described in table 1. We use leading order formulæ for the widths and cross section [13],since the leading QCD corrections factorize out in the ratio. In the 


\begin{tabular}{|c|c|}
\hline$m_{h}<131.5$ & $R_{\gamma \gamma}=\frac{\sigma_{g g \rightarrow h}}{\sigma_{g g \rightarrow h}^{(\mathrm{SM})}} \cdot \frac{\mathrm{BR}_{h \rightarrow \gamma \gamma}}{\mathrm{BR}_{h \rightarrow \gamma \gamma}^{(\mathrm{SM})}}$ \\
\hline $131.5<m_{h}<2 m_{W}$ & $R_{W W^{*}}=\frac{\sigma_{g g \rightarrow h}}{\sigma_{g g \rightarrow h}^{(\mathrm{SM})}} \cdot \frac{\mathrm{BR}_{h \rightarrow W W^{*}}}{\mathrm{BR}_{h \rightarrow W W^{*}}}$ \\
\hline$m_{h}>2 m_{W}$ & $R_{V V}=\frac{\sigma_{g g \rightarrow h}}{\sigma_{g g \rightarrow h}^{(\mathrm{SM})}}$ \\
\hline
\end{tabular}

Table 1. Higgs cross section times branching ratio normalized to the SM value for the different intervals of Higgs masses used in the analysis.

cases we study, the vector boson fusion channel is always subdominant compared to the gluon-gluon fusion one for the Higgs production cross section and can be safely neglected.

We also implement LHC bounds using the latest results from Higgs searches with $\sim 5 \mathrm{fb}^{-1}$ [19-25]. In particular, for each Higgs mass, we compare the quantities in table 1 with the strongest between the ATLAS and the CMS bounds on the $h \rightarrow \gamma \gamma$ channel for $m_{h}<131.5 \mathrm{GeV}$, the CMS bounds on $h \rightarrow W W^{*}$ for $131.5 \mathrm{GeV}<m_{h}<2 m_{W}$ and the CMS combination of $h \rightarrow W W$ and $h \rightarrow Z Z$ for $m_{h}>2 m_{W}$, respectively. The accuracy of such bounds should be taken with a grain of salt, because of the uncertainties both on the theory side for the Higgs mass calculation and on the experimental side for the energy scaling calibration. For example, a shift of order $1-2 \mathrm{GeV}$ in energy can either amplify or dump sensibly the bounds around $125 \mathrm{GeV}$ where both ATLAS and CMS have excesses.

Given the sensitivity of $b \rightarrow s \gamma$ to $m_{A}, m_{\tilde{t}_{2}}$, and $A_{t}$, we calculate the corresponding bounds separately in each example using the SUSYBSG code [37] for light $(600 \mathrm{GeV})$ and heavy $(10 \mathrm{TeV})$ squarks of the first and second family.

Finally for estimating the fine-tuning we use eq. (2.1). In the MSSM and DMSSM cases the main contributions come from the $\mu$-term at tree level and from $m_{\tilde{t}_{i}}$ and $A_{t}$ at one loop. We set the mediation scale to $100 \mathrm{TeV}$ to minimize the tuning. For the NMSSM we also consider the contributions coming from the singlet sector.

In all cases, the region with very large stop mixing is disfavored by vacuum instabilities and/or large corrections to the $\rho$ parameter. Most of this region has been crossed out in the plots by requiring the Higgs to be above the LEP bounds.

For our calculation we use the following parameters without loss of generality

\begin{tabular}{|c|c|c|c|c|c|}
\hline$m_{t}$ & $M_{2}$ & $M_{3}$ & $m_{\tilde{t}_{L}}^{2}-m_{\tilde{t}_{R}}^{2}$ & $m_{\tilde{b}_{R}}$ & $\Lambda$ \\
\hline 173.2 & 300 & 1000 & $100^{2}$ & $\infty$ & $10^{5}$ \\
\hline
\end{tabular}

In particular gauginos masses, whose impact on the quantities we discuss is marginal, are heavy enough not to be ruled out but light enough not to affect the fine-tuning. The left- and right- hand stop masses are almost degenerate and we have checked that a larger splitting between these soft masses increases only the fine-tuning without altering the conclusion of our analysis. The role of the sbottoms is marginal, and we keep the left-handed sbottom while the other is decoupled. 


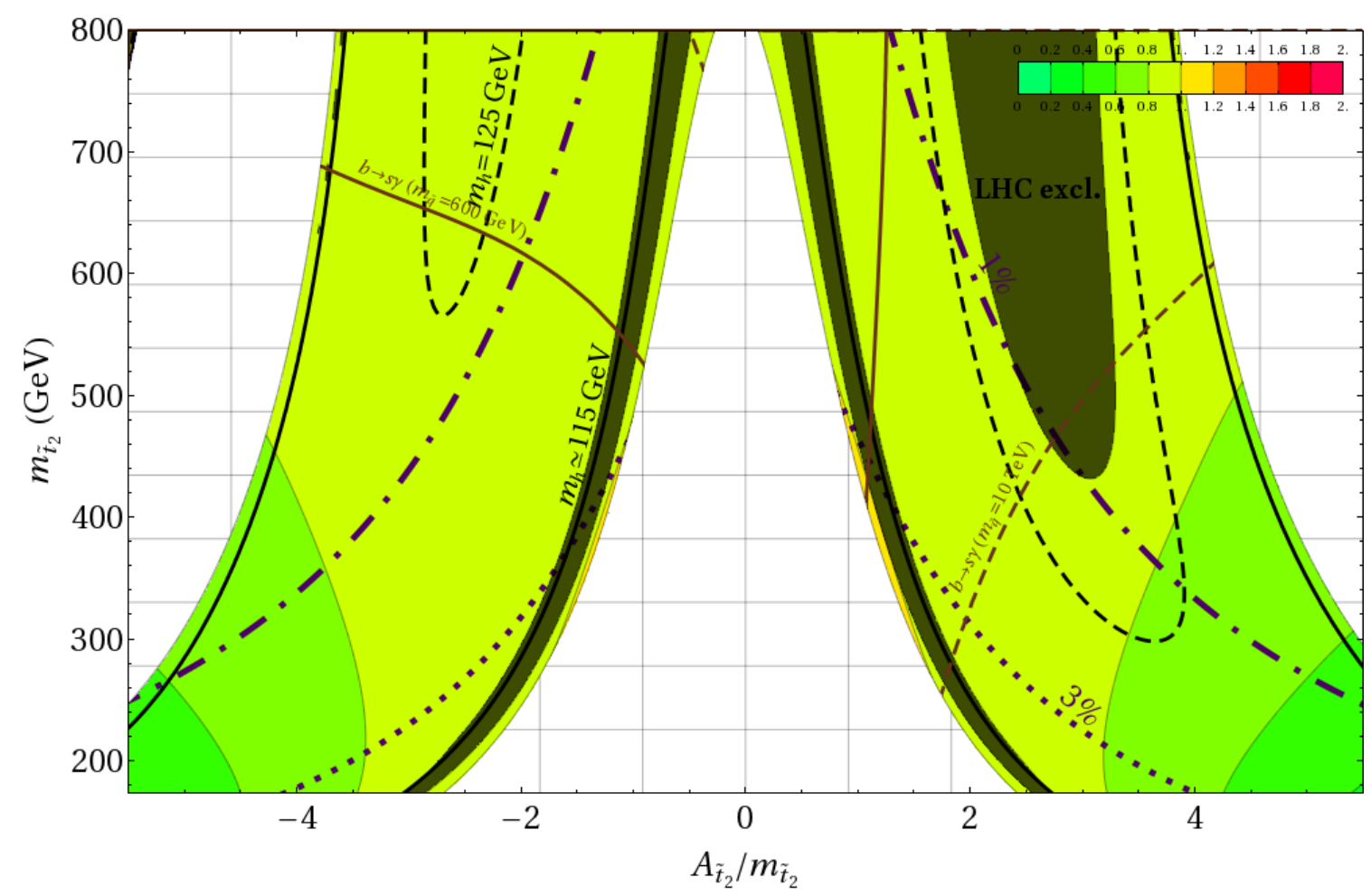

Figure 3. We present Higgs production contours relative to the SM in the MSSM scenario for $m_{A}=800 \mathrm{GeV}$. We block out the region where the Higgs is below the LEP bound. We also draw the contours of $1 \%$ (dotted-dashed)and $3 \%$ (dotted) tuning. The region allowed by $b \rightarrow s \gamma$ at $95 \% \mathrm{CL}$ lies above the solid (dashed) line when squarks are at $600 \mathrm{GeV}(10 \mathrm{TeV})$. The shaded region is instead excluded by the $\mathrm{LHC}$.

We follows the conventions in [38] except for the $\mu$-term, which we define with the opposite sign, and the ordering of the stop eigenvalues, which we choose to be $m_{\tilde{t}_{2}}<m_{\tilde{t}_{1}}$. For the singlet sector of the NMSSM we instead use the conventions of [36] .

\subsection{The Higgs in the MSSM}

In this class of models we consider the minimal SUSY spectrum and we relax the requirement of universality for the scalar masses allowing only the stops to be light. With no additional contributions to the Higgs mass in the MSSM, the radiative corrections from the stop sector are the only way to satisfy the LEP bound. The region where the tuning is minimized and the Higgs is still heavy enough is the one with large stop mixing and $\tan \beta$ [39]. In this region of parameter space, the Higgs is light and the most relevant channel at the LHC is $h \rightarrow \gamma \gamma$, thus in figures 3,4 , and 5 only $R_{\gamma \gamma}$ has been used.

For the plots we choose $\mu=200 \mathrm{GeV}$, and $\tan \beta=10$, to allow for the Higgs to lie above the LEP bounds, but not too large to avoid stronger bounds from direct and indirect searches. 


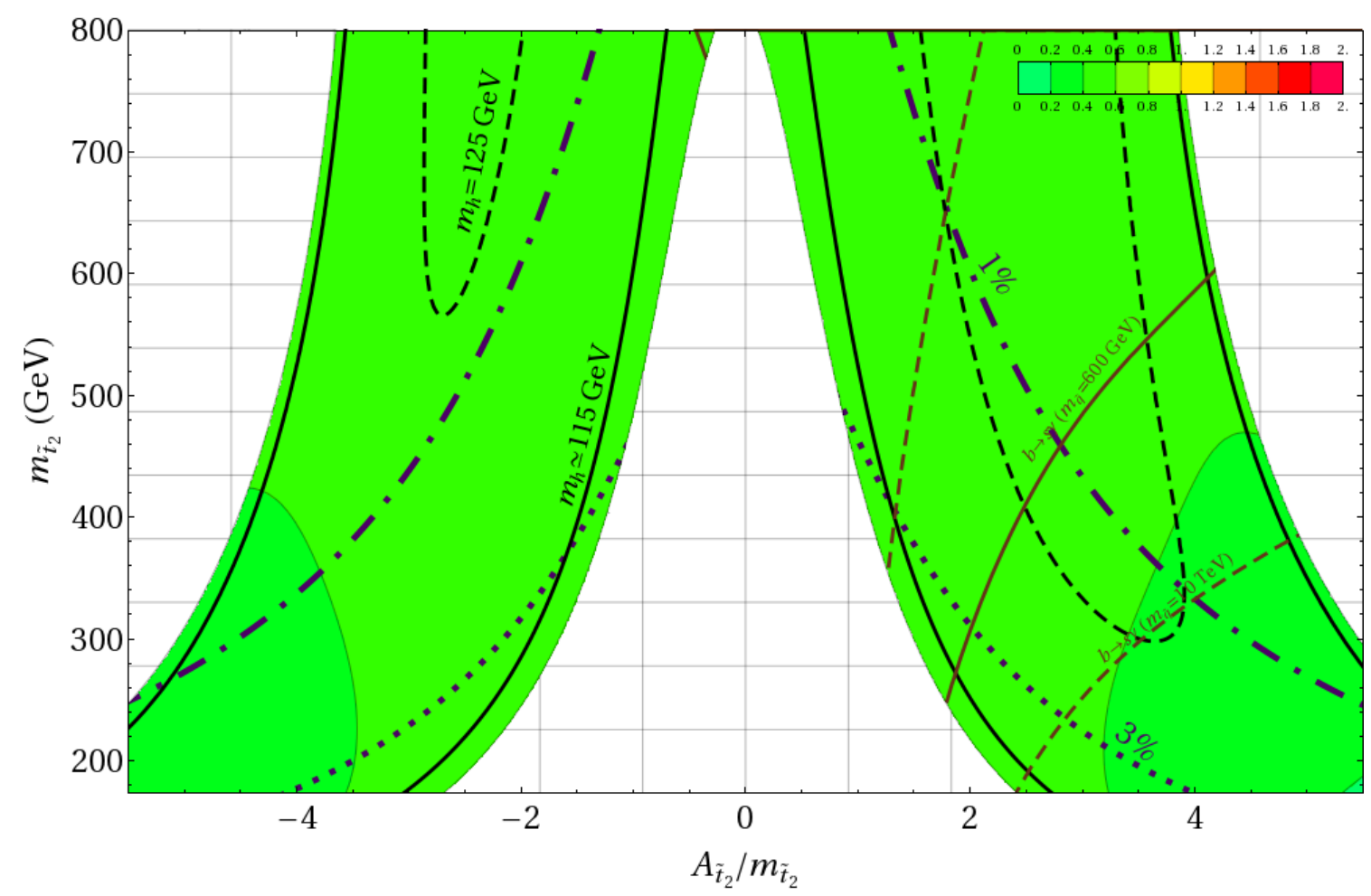

Figure 4. Same as figure 3 , but with $m_{A}=250 \mathrm{GeV}$.

In figure 3 we show how $R_{\gamma \gamma}$ varies as a function of the lightest stop mass and the ratio of the $A$-term over the stop mass, when $m_{A}=800 \mathrm{GeV}$. Now, the tree level effects from the Higgs mixing are negligible. We block out the region where the Higgs is below the LEP bound. The Higgs mass increases to $\sim 120(\sim 130) \mathrm{GeV}$ in the inner part of the allowed region for low(large) values of the stop mass. The asymmetry of the Higgs mass contours with respect to the stop mixing arises from a two loop gluino-stop correction and is correlated with the relative sign of $A_{t}$ and the gluino mass. ${ }^{2}$ We also show the contours of $1 \%$ and $3 \%$ tuning, which has been estimated through eq. (2.1). With a large $\tan \beta$ and small $\mu$-term, the main contribution to the tuning comes from the radiative stop corrections, as expected. Since $m_{A}$ is large, loop effects are responsible for changing the Higgs production. LEP bounds on the Higgs mass select the region of large $A_{t}$, where $R_{\gamma \gamma}$ gets suppressed. The suppression is however only large for very low stop masses, which are incompatible with $b \rightarrow s \gamma$ bounds. The latter push us to the decoupling limit, where the Higgs looks more SM-like, with a $\sim 20 \%$ reduction of its production cross-section at best. Low fine-tuning though in this case still favors the presence of one stop at around $400 \mathrm{GeV}$, which could be within the reach of the $7 \mathrm{TeV}$ LHC. Finally we can also see how the LHC started probing this low Higgs mass region: masses around $115 \mathrm{GeV}$ have just been ruled out by ATLAS, while masses above $127 \mathrm{GeV}$ from CMS.

\footnotetext{
${ }^{2}$ We thank P. Slavich for clarifying this point.
} 


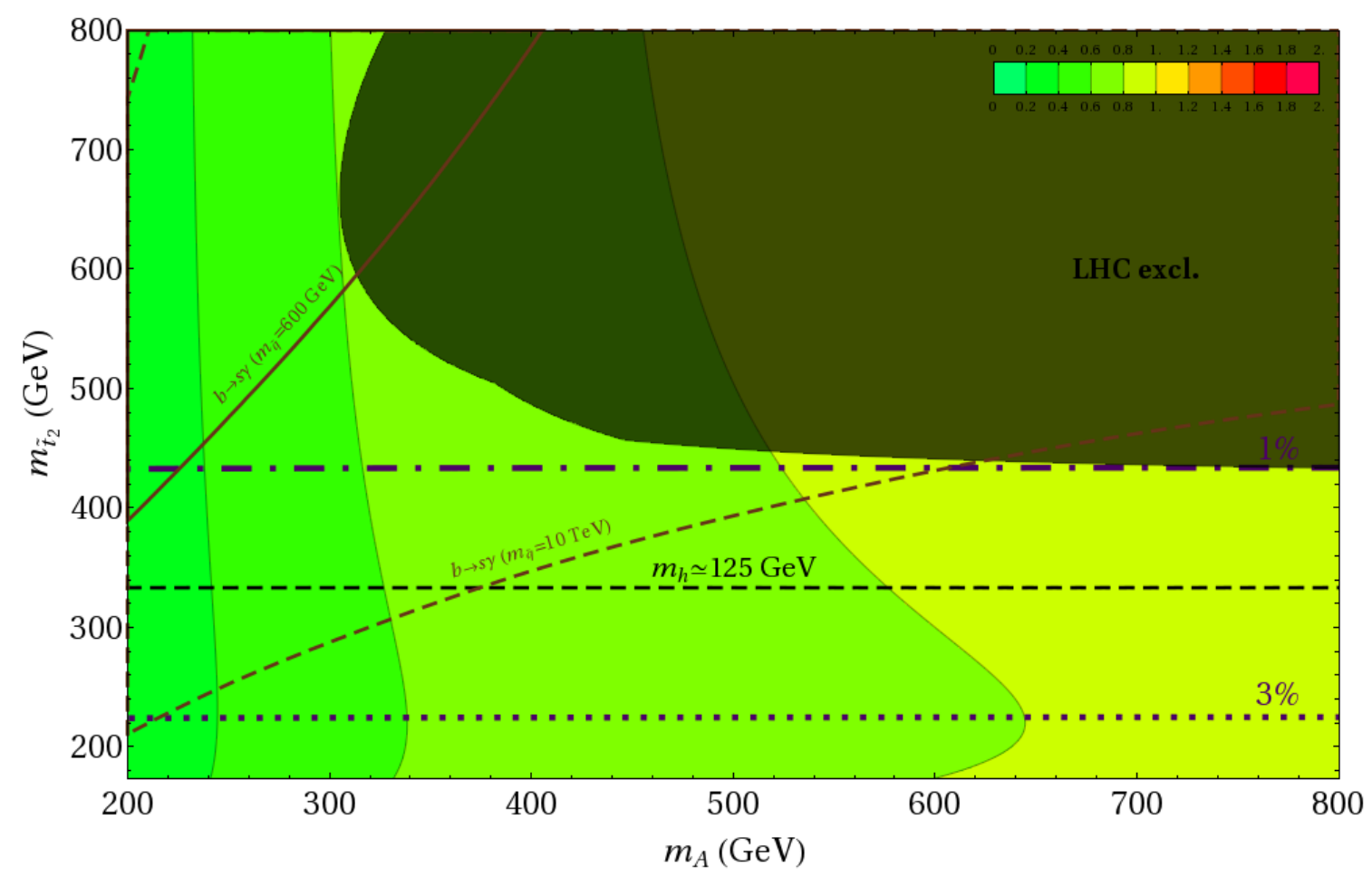

Figure 5. Higgs production relative to the SM as a function of the $m_{A}$ and the lightest stop mass when $A_{t}=3 m_{\tilde{t}_{2}}$ in the MSSM case. We also present the same Higgs mass, fine-tuning and $b \rightarrow s \gamma$ contours as in figure 3 .

In figure $4 m_{A}$ is now lowered to $250 \mathrm{GeV}$, Higgs mixing effects become important and $\mathrm{BR}(h \rightarrow \gamma \gamma)$ is suppressed compared to its SM value because $h \rightarrow b \bar{b}$ is enhanced. At the same time, pushing the Higgs mass above the LEP bound requires large $\frac{A_{t}}{m_{\tilde{t}_{2}}}$, further suppressing $R_{\gamma \gamma}$, which is now roughly around half the SM value in most of the allowed parameter space. In this case, the Higgs remains hidden at the LHC, probably till the end of next year. It is worth pointing out, however, that the requirement of small tuning favors the presence of a light stop at around $300 \mathrm{GeV}$ which may show up before the Higgs is discovered.

In figure 5 , keeping $A_{t}=3 m_{\tilde{t}_{2}}$, we show how the shape of the contours changes by increasing $m_{A}$ signaling the decoupling of the tree-level effects from Higgs mixing. Higgs mixing effects can easily provide a $40 \%$ suppression of $g g \rightarrow h \rightarrow \gamma \gamma$ compared to the SM, but they become unimportant for $m_{A}>400 \mathrm{GeV}$ as expected, since we are well within the decoupling limit of the Higgs sector. Figure 5 also shows that the fine-tuning is insensitive to $m_{A}<1 \mathrm{TeV}$ because its contribution to the fine-tuning is suppressed by $\tan \beta$, as discussed previously. $b \rightarrow s \gamma$ bounds push towards heavier stop masses (and Higgs masses as well), unless $m_{A}$ is light enough to allow for a cancellation between the charged Higgs and the stop-chargino loops.

In conclusion we see that the Higgs is always light in the minimal implementation of supersymmetry. In the less tuned region, the requirement of large $A_{t}$ in order to push the 
Higgs mass above the LEP bound guaranties that $R_{\gamma \gamma}$ will always be smaller than one. This suppression is enhanced further when the pseudoscalar Higgs is light and the Higgs can now be hidden from the LHC till the end of next year.

\subsection{The Higgs in the MSSM with extra D-terms}

In these models, the SM gauge sector is extended with an extra gauge group that is broken not much above the weak scale and under which the Higgs is charged [26]. When the field responsible for the breaking of that group gets a soft mass around the weak scale, the D-term for the Higgs results in an increase of the Higgs quartic and the tree level mass of the Higgs gets an additional contribution. We explore this scenario assuming that the D-term is generated by a $\mathrm{U}(1)_{x}$ symmetry under which $h_{u}$ and $h_{d}$ are vector-like and the new contribution to the Higgs potential is given by

$$
\delta V=\frac{\lambda_{x}^{2}}{8}\left(h_{u}^{2}-h_{d}^{2}\right)^{2} .
$$

We choose $\lambda_{x}=0.7$, large enough to allow the Higgs mass to lie confortably above the LEP bounds without the need of $A$-terms but low enough such that the corresponding $\mathrm{U}(1)_{x}$ can satisfy electroweak precision tests and collider searches [40]. We keep the rest of the parameters for the charginos and the sbottoms the same as in the MSSM case (see section 3.1) but change $\tan \beta$ to 5 . We now find that the Higgs easily is above the LEP bound in the entire region of the parameter space we are considering. According to table 1 , since the Higgs mass never exceeds $2 m_{W}$, in figure 6 , 7, and 8, we show $R_{\gamma \gamma}$ when the Higgs is between the LEP bound and $131.5 \mathrm{GeV}$, and $R_{W W^{*}}$ when the Higgs is above $131.5 \mathrm{GeV}$.

In figure 6 we show $R_{\gamma \gamma}$ and $R_{W W^{*}}$ as a function of the lightest stop mass and the ratio

$\frac{A_{t}}{m_{\tilde{t}_{2}}}$ for $m_{A}=800 \mathrm{GeV}$. The fine-tuning is significantly relaxed due to the extra tree-level contribution to the Higgs mass and we now plot the contours of 5\% and $15 \%$ fine-tuning. The lightest Higgs state can now be heavier, and loop effects become more pronounced in the decoupling limit of the heavy Higgs sector. Since the region $A_{t} \sim 0$ is not excluded anymore, there is the possibility of $R_{\gamma \gamma}>1$ provided that bounds from $b \rightarrow s \gamma$ are satisfied. This is possible when the rest of the squarks are light $(\sim 600 \mathrm{GeV})$, otherwise the Higgs is pushed to a region when it looks more SM-like. In this case, $b \rightarrow s \gamma$ seems to constrain the hierarchy between the third and the other two families in split family scenarios when a gauge mediation mechanism is present and the $A$-terms are small. When we apply the latest LHC Higgs search results, we see that most of the parameter space is now excluded leaving only open the possibility of very light stops and small $A$-terms where the Higgs production cross-section is enhanced up to $50 \%$. Note that this region is still allowed in the plot because of the ATLAS and CMS excesses around $125 \mathrm{GeV}$.

In figure 7 , we present the same parameter space when $m_{A}=250 \mathrm{GeV}$. Similarly to the MSSM case, the effects of the light pseudoscalar mass are evident everywhere in the parameter space, due to the increase of the $h \rightarrow b \bar{b}$ width, but now the suppression can be smaller as small $A$-terms are allowed. Rare $\mathrm{B}$ decays now exclude the possibility of zero $A$-terms and further constrain split family scenarios when a gauge mediation mechanism is considered. 


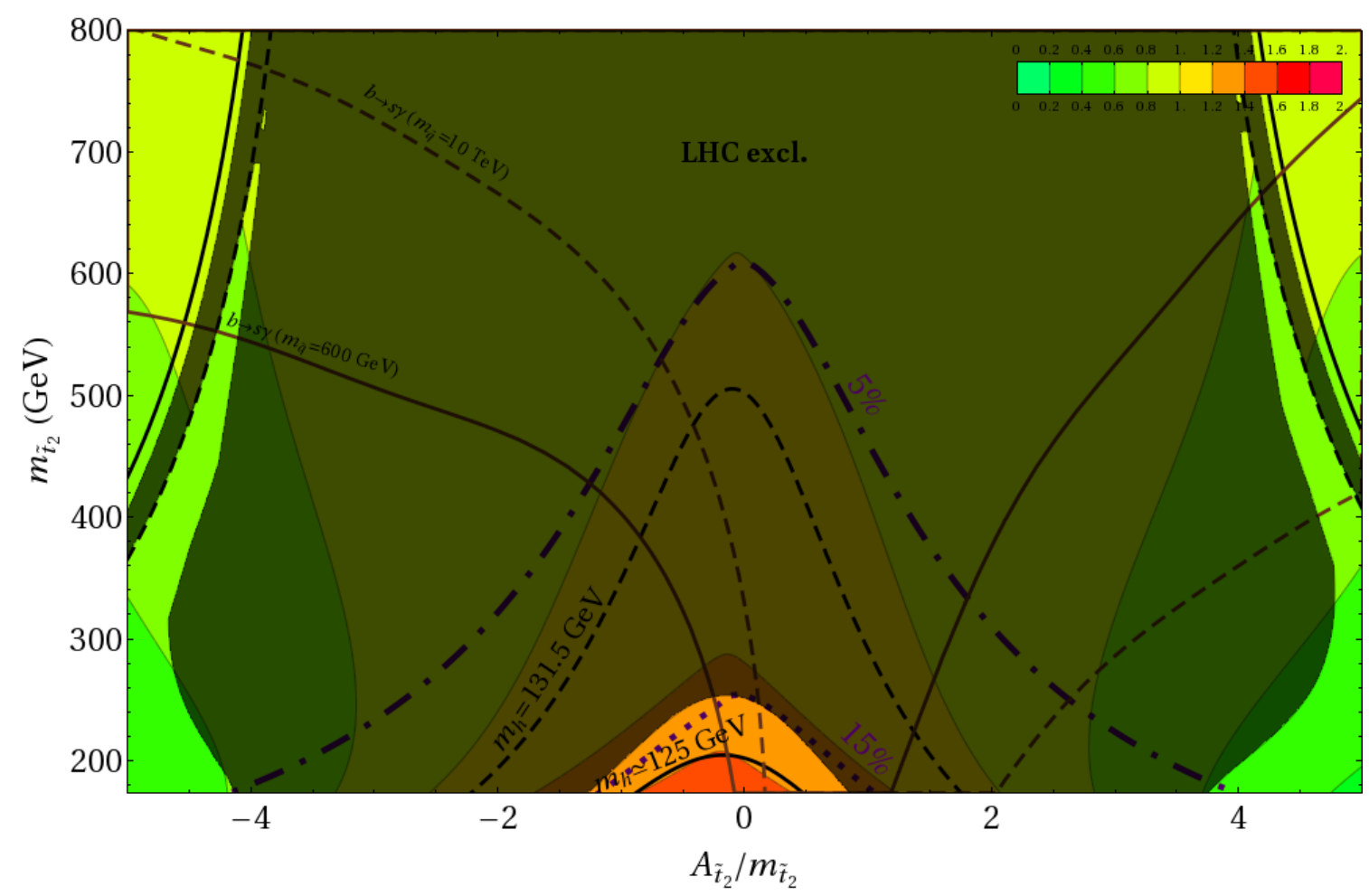

Figure 6. Higgs production at the LHC compared to the SM when Higgs D-terms are added to the MSSM with $m_{A}=800 \mathrm{GeV}$ in the $A_{t} / m_{\tilde{t}_{2}}-m_{\tilde{t}_{2}}$ plane. We show the contours of $15 \%$ (dotted) and $5 \%$ (doted-dashed) fine-tuning as well as the Higgs mass contours of $125 \mathrm{GeV}$ and $131.5 \mathrm{GeV}$.We also show the regions allowed by $b \rightarrow s \gamma$ at $95 \%$ CL and the shaded region ruled out by the LHC, as in the case of the MSSM.

Finally in figure 8 we show explicitly the dependence on $m_{A}$, keeping fixed $A_{t}=0$, which is the value preferred by low scale SUSY-breaking scenarios, such as gauge (GMSB) and anomaly mediation (AMSB). The slight difference between $R_{\gamma \gamma}$ and $R_{W W^{*}}$ across the 131.5 GeV Higgs mass contour is due to Higgs mixing effects suppressing the Higgs coupling to $W W$ less than the Higgs coupling to tops. The overall width $h \rightarrow \gamma \gamma$ gets a small boost in this region because the top contribution now subtracts less from the $W$ contribution and $R_{\gamma \gamma}$ tends to be a bit higher than $R_{W W^{*}} . b \rightarrow s \gamma$ bounds push either the stop or $m_{A}$ to be heavy, with a preference for light 1st and 2nd generation squarks. After LHC bounds are taken into account, only two regions are singled out, one more tuned with large stop masses and reduced coupling of the Higgs (due to Higgs mixing) and one with light stops, heavy $m_{A}$ and enhanced $R_{\gamma \gamma}$. A SM-like Higgs in this scenario is very unlikely.

In conclusion, in this kind of models generically the Higgs cross section and branching ratios differ from the SM even by order one. Strong bounds from the LHC apply and when combined with bounds from $b \rightarrow s \gamma$ and naturalness they strongly constrain the allowed parameter space, especially in scenarios with vanishing squark mixing. 


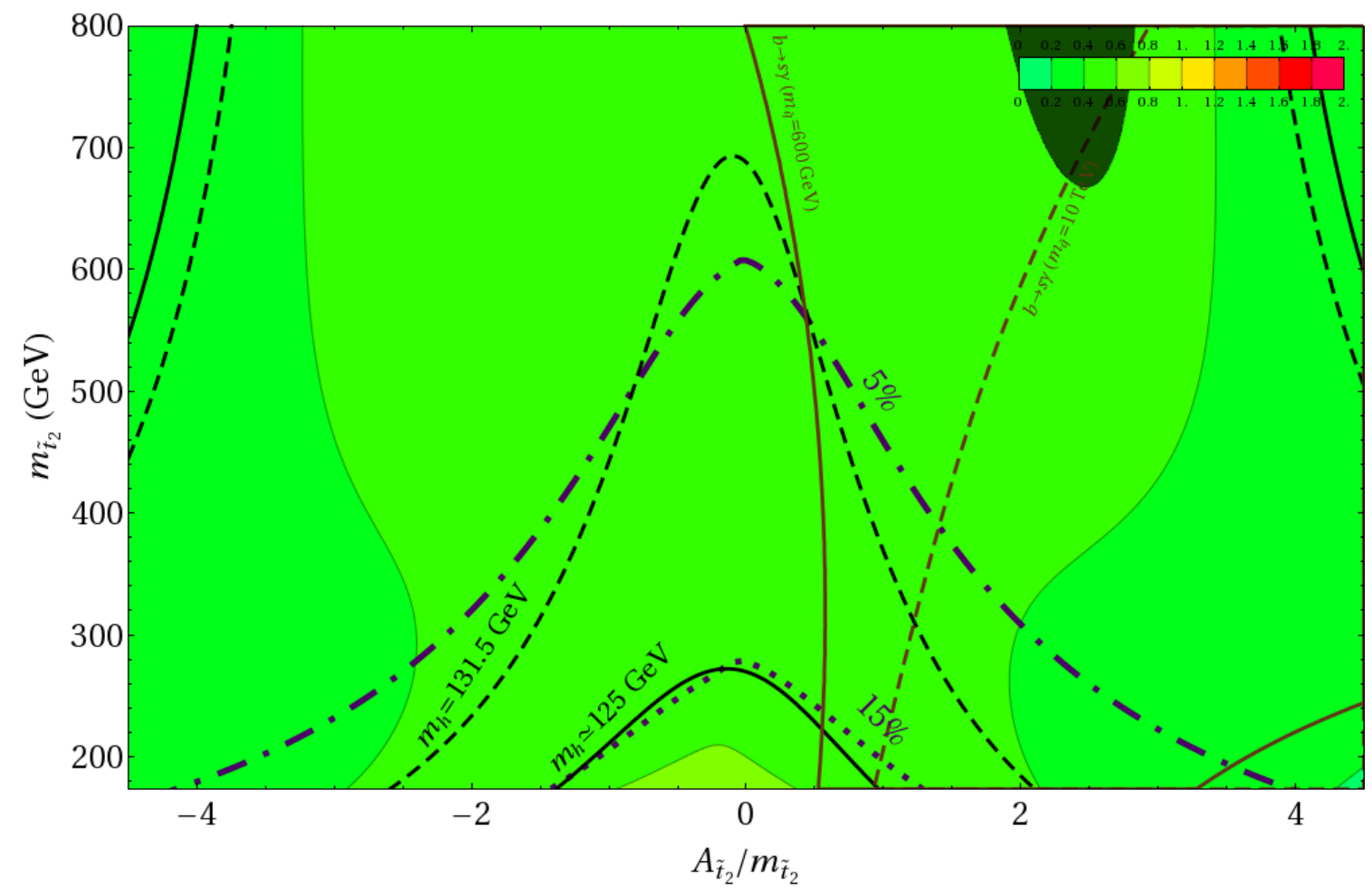

Figure 7. Same as figure 6 but with $m_{A}=250 \mathrm{GeV}$.

\subsection{The Higgs in the NMSSM}

Motivated by the $\mu$ problem, the NMSSM extends the Higgs sector by a singlet that now mixes with the MSSM Higgses. We consider the $Z_{3}$ symmetric version of the NMSSM with the following superpotential:

$$
W=\lambda S H_{u} H_{d}-\frac{\kappa}{3} S^{3}
$$

Symmetry breaking in the singlet sector strongly depends on the singlet $A$-terms, $\lambda A_{\lambda} S H_{u} H_{d}$ and $\kappa A_{\kappa} S^{3} / 3$, which have to be non-zero for the singlet to acquire a vev. There are also requirements on these terms from vacuum stability [27, 41].

We select two qualitatively different regions of the NMSSM parameter space (in $\mathrm{GeV}$ )

\begin{tabular}{|c|c|c|c|c|c|}
\hline$\mu=\lambda v_{s}$ & $\tan \beta$ & $\lambda$ & $\kappa$ & $A_{\lambda}$ & $A_{\kappa}$ \\
\hline 200 & 1.5 & 0.7 & -0.7 & 750 & -750 \\
\hline 300 & 1.4 & 1.4 & -1.4 & 1000 & -1000 \\
\hline
\end{tabular}

The first we refer to as perturbative NMSSM, see figure 9, the second as $\lambda S U S Y$, see figure 10. Both regions are chosen such that there are no pseudoscalar Higgs states for the light scalar Higgs to decay into that can drastically alter the Higgs width. Indirect bounds from $b \rightarrow s \gamma$ are now irrelevant since at small $\tan \beta$ the SUSY contributions are smaller and they always tend to cancel (see figure 2). 


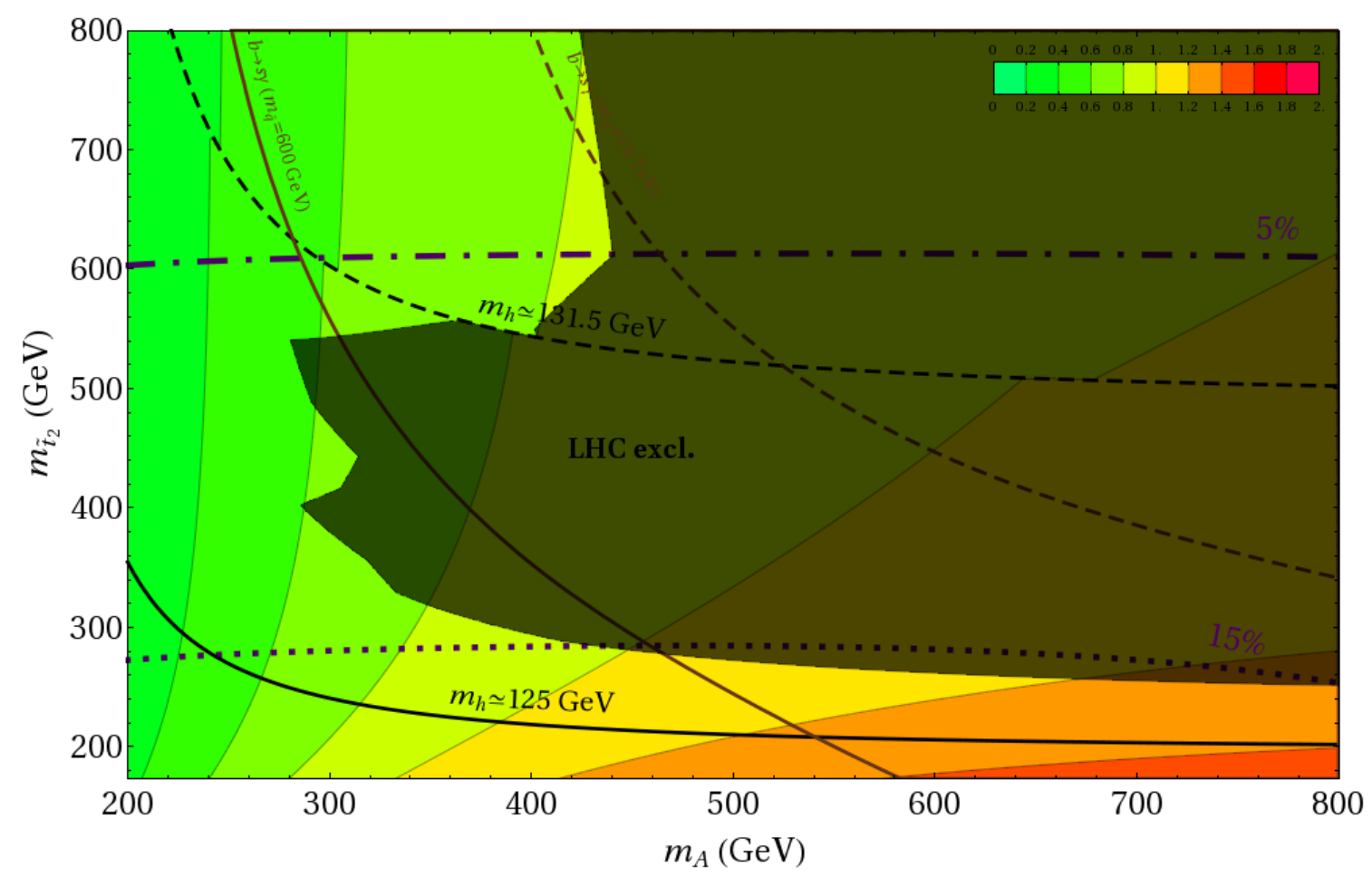

Figure 8. Higgs production relative to the SM as a function of the $m_{A}$ and the lightest stop mass when $A_{t}=0$ in the MSSM with D-terms. We also present the same Higgs mass, $b \rightarrow s \gamma$ and fine-tuning contours as in figure 6 .

In the perturbative NMSSM, we choose small values of the singlet couplings that can remain perturbative up to the GUT scale. The value of $\tan \beta$ now is small in order to maximize the contributions to the Higgs mass. The Higgs mass takes values up to $150 \mathrm{GeV}$. In figure 9 , we present $R_{\gamma \gamma}$ and $R_{W W^{*}}$ as a function of $m_{\tilde{t}_{2}}$ and $A_{t} / m_{\tilde{t}_{2}}$. We can easily see that mixing effects in the Higgs sector are very suppressed as $\tan \beta$ is small and $h \rightarrow b \bar{b}$ does not change a lot compared to its SM value. In addition, the preferred $A$-terms are small and the Higgs starts being overproduced in a large part of the parameter space; the Higgs is slightly heavier than the previous two cases and loop effects decouple more slowly with increasing stop mass. In fact, current LHC searches highly constrain a big part of this parameter space, leaving out the regions with $m_{h} \lesssim 130 \mathrm{GeV}$, where the bounds are weaker. In particular the region near $A_{t} \sim 0$ is still allowed despite the enhancement because of the excess around $125 \mathrm{GeV}$ in the ATLAS and CMS results. The other region which survives is the one with very large $A$-terms where the Higgs production cross section is sufficiently suppressed. Most of these regions of the parameter space should be covered by the end of next year.

In $\lambda S U S Y[28,29]$, the perturbativity condition at $M_{G U T}$ on the couplings is relaxed, with virtually no bounds on the value of the Higgs mass. For the parameters we have chosen, the Higgs becomes as heavy as $\sim 250 \mathrm{GeV}$. In most of the parameter space $m_{h}>2 m_{W}$ and we only plot $\sigma \times B R\left(h \rightarrow W W^{(*)}\right)$. The mixing in the Higgs sector is not as important because the Higgs decay to $b \bar{b}$ no longer dominates the Higgs width. Loop effects become more 


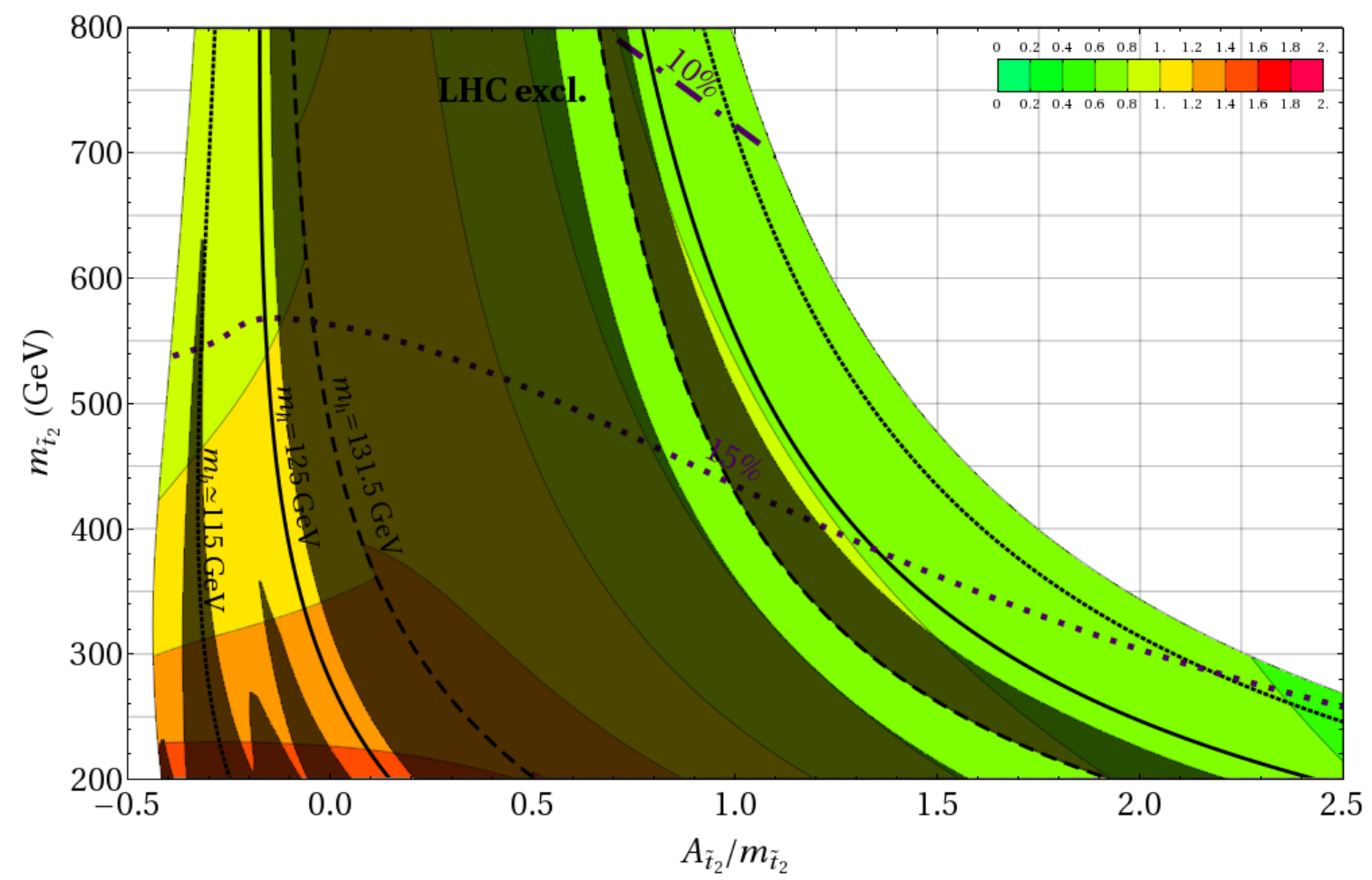

Figure 9. We present the contours of Higgs production relative to the SM in the perturbative NMSSM scenario. We draw the contours of $10 \%$ and $15 \%$ tuning. We also show the 115,125 and 131.5 GeV Higgs mass contours. The dark shaded area has been excluded by LHC Higgs searches.

important for heavier stops due to the large Higgs mass, and the Higgs is overproduced for a large part of the parameter space, when the $A$-terms are not too large [42]. The strength of the LHC bounds for a heavy Higgs together with this enhancement of the cross section make most of the parameter space excluded. The only region still allowed is the one with small Higgs mass, near the boundary of stability, where there is an enhancement due to large mixings with the heavier Higgs states. We will return on this particular region in section 3.4. In this region we still plot $\sigma \times B R\left(h \rightarrow W W^{*}\right)$ because this channel is enhanced with respect to the diphoton one in this model and, even though the LHC bounds from $h \rightarrow \gamma \gamma$ are stronger than those from $h \rightarrow W W^{*}$ for a SM Higgs, the former are not constraining in this case.

It is fair to say that $\lambda S U S Y$ is highly disfavored by LHC searches, although corners of parameter space are still allowed, as well as the possibility to hide the Higgs through decay to light pseudoscalars.

\subsection{Large mixing between Higgs states}

\subsubsection{Mixing in the MSSM}

We discuss now the case of large mixing between the light and the heavy Higgses in the MSSM, corresponding to values of $m_{A} \gtrsim 100 \mathrm{GeV}$ and $\tan \beta \lesssim 10$, still allowed experimentally (see figure 1). In this special region, the light and the heavy Higgs do not have a 


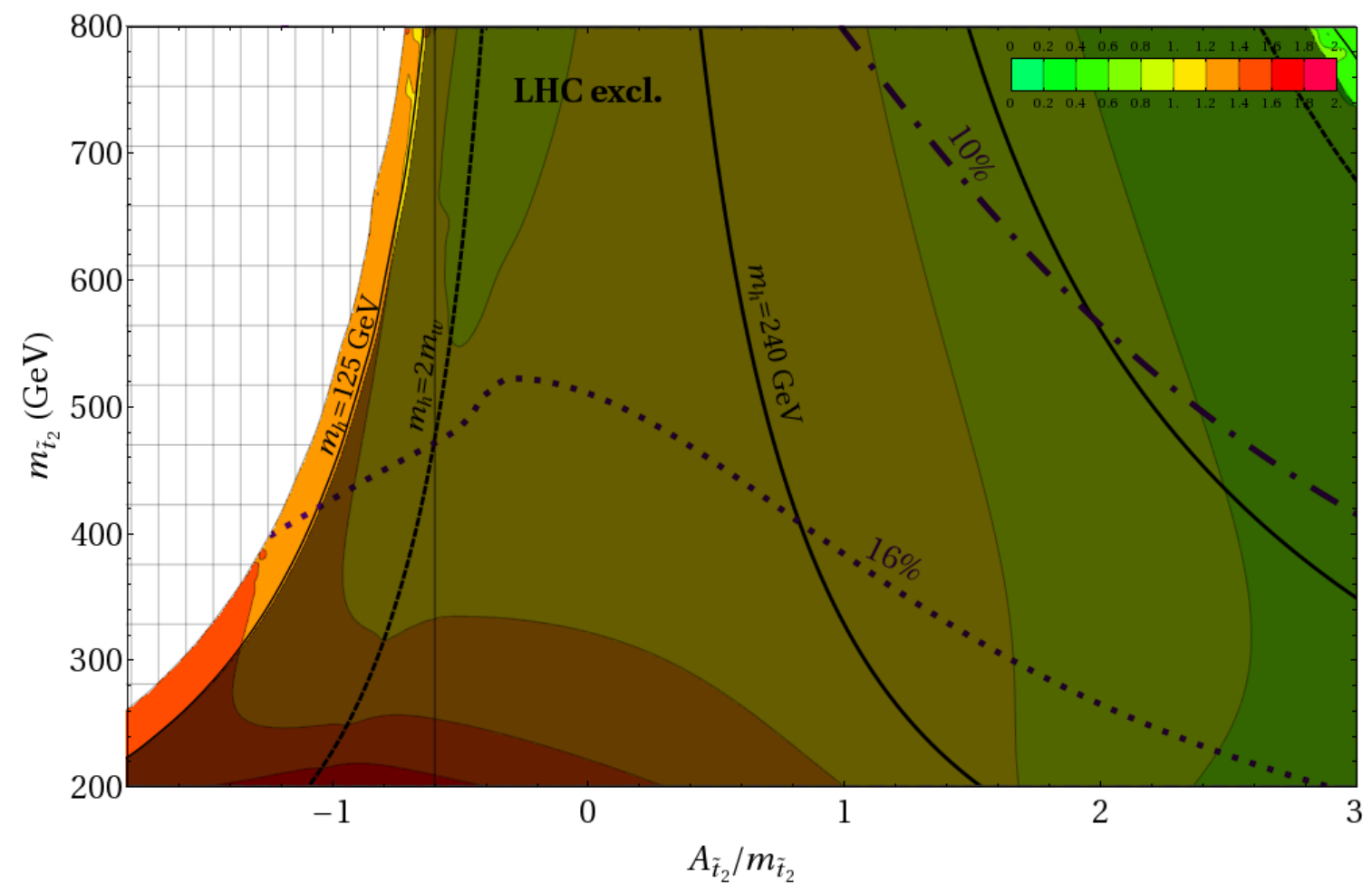

Figure 10. We present the contours of $\sigma \times B R\left(h \rightarrow W W^{(*)}\right)$ relative to the SM in the $\lambda S U S Y$ scenario. We draw the contours of $10 \%$ and $16 \%$ tuning. We also show the $125,2 m_{W}$, and $240 \mathrm{GeV}$ Higgs mass contours. The dark shaded area has been excluded by LHC Higgs searches.

large mass splitting and the role of the SM Higgs is almost equally shared between the two states. The behavior of the Higgses strongly depends on their mass. As already discussed at the beginning of section 2, while the couplings to the top and the $W$ are both suppressed compared to their SM values, the coupling to the bottom is enhanced. The production of both Higgs states at the LHC can be highly suppressed, when the $h \rightarrow \gamma \gamma$ and $h \rightarrow W W^{*}$ channels are relevant. At large $\tan \beta$, the pseudoscalar Higgs may signal the presence of an extended Higgs sector at the LHC, since its couplings are not affected by the mixing and are enhanced by $\tan \beta$. When the width is no longer dominated by decays to $b \bar{b}$, we expect one Higgs to be visible, when the cross-section of the other Higgs is suppressed, given that

$$
y_{t \bar{t} h}^{2}+y_{t \bar{t} H}^{2}=\frac{1}{\sin ^{2} \beta},
$$

where $y_{t \bar{t} h}$ and $y_{t \bar{t} H}$ are the top couplings to the light and heavy Higgs, respectively.

In figure 11(a) and (b), we show an example for the light and the heavy Higgs contours of $R_{\gamma \gamma}$ in the MSSM scenario. The parameters used are the same as in section 3.1 except for $\tan \beta$ which we set to 8 and $m_{A}=100 \mathrm{GeV}$.

When the Higgs mass is below $\sim 114 \mathrm{GeV}$, LEP searches are the most sensitive and we substitute $R_{\gamma \gamma}$ for the ratio of the Higgs coupling to the $W$

$$
R_{W W^{*}}^{h}=\sin ^{2}(\beta-\alpha),
$$


(a)

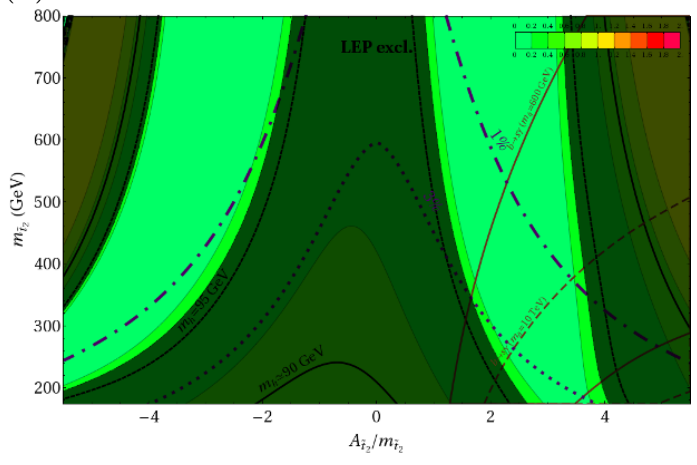

(b)

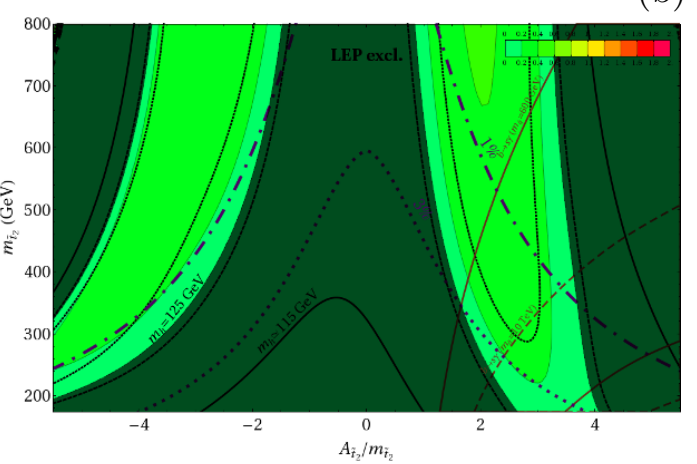

Figure 11. Contours of Higgs production relative to the SM when the Higgs maximally mix in the MSSM. We show tuning contours (dotted and dashed-dotted), $b \rightarrow s \gamma$ allowed regions between the solid (dashed) for light (heavy) squarks, and different Higgs mass contours. Finally, the dark shaded areas are excluded by Higgs searches.

$$
R_{W W^{*}}^{H}=\cos ^{2}(\beta-\alpha)
$$

We also show the excluded region from LEP bounds on the light Higgs, which significantly constrain the parameter space for the heavy Higgs as well. For the parameter space still not probed, the increase in $h \rightarrow b \bar{b}$ for both Higgses hides them at the LHC, but the pseudoscalar Higgs is light enough and it is well within the reach of the next round of $A \rightarrow \tau^{+} \tau^{-}$searches at the LHC, as can be seen in figure 1 .

The scenario with additional D-terms for the Higgs differs in that the heavy Higgs can be heavy enough for decays to $b \bar{b}$ to be irrelevant and its LHC signals are enhanced. As a result, a lot of the parameter space in this case can be constrained from the absence of the heavy Higgs.

\subsubsection{Mixing in the NMSSM}

In the NMSSM, the addition of the singlet affects the Higgs couplings to the SM particles in two ways. First, it suppresses all Higgs couplings by directly mixing with $H_{u}$ and $H_{d}$. Another effect comes from the $S H_{u} H_{d}$ coupling contribution to the Higgs quartic in the F-term potential. Analogously to the DMSSM case, the effects of this quartic do not decouple when the singlet gets its mass from SUSY breaking. This new contribution is always positive contrary to the MSSM where it is generated by D-terms. ${ }^{3}$ The top (bottom) Yukawa is now enhanced (suppressed) compared to its SM value, opposite to what is happening in the MSSM as seen in eq. (2.6). For a light Higgs, this leads to an enhancement of $R_{W W^{*}}$ because the Higgs width to bottoms decreases.

These two effects mentioned above combine in the left side of figure 10 to enhance the Higgs $\sigma \times B R$. In this region, even though the singlet and the heavy Higgs are always above $400 \mathrm{GeV}$ there are still large mixing effects because the lightness of the Higgs is driven by cancellations in the $3 \times 3$ Higgs mass matrix. For the same reason, the heavy Higgs, which

\footnotetext{
${ }^{3}$ We thank R. Rattazzi for pointing this out.
} 
is around $400 \mathrm{GeV}$, has non negligible couplings to vector bosons and now LHC searches for a heavy SM Higgs may place the strongest bound on this region.

\subsection{Higgs coupling determination at the LHC}

It is obvious that deviations of the Higgs from the SM behavior may signal the presence of new light degrees of freedom. But how well can the LHC measure these deviations after the Higgs is discovered? QCD uncertainties in the Higgs production introduce $10-20 \%$ error in the measurement of $\sigma \times B R$ for every Higgs decay channel. In most cases, absolute coupling determination cannot be better than $20 \%[43,44]$. These uncertainties though disappear when considering the ratios of $\sigma \times B R$ for channels that have the same production mechanism. In this case, most systematics are of the order of a few $\%$ and it is possible for the LHC to become a precision probe of Higgs couplings. An example of such a ratio for a Higgs below $\sim 130 \mathrm{GeV}$ is:

$$
\frac{\sigma \times B R(h \rightarrow \gamma \gamma)}{\sigma \times B R\left(h \rightarrow Z Z^{*}\right)} .
$$

For both channels, energy resolution is excellent and LHC will have enough statistics to eventually be systematics limited. This ratio in the MSSM becomes sensitive to stop loop effects which can be as large as $50 \%$ in the large mixing region. For example, an accuracy of $3 \%$ in such ratio may probe stop masses as high as $500 \mathrm{GeV}$. The ratio becomes sensitive to mixing effects as well, when $\tan \beta$ is small. This ratio can be enhanced compared to the SM value only when stop mixing is large or the top coupling is suppressed by mixing effects. In this case, the Higgs production cross-section will also be suppressed.

Another possible ratio is the photon channel compared to the $\tau^{+} \tau^{-}$(or $b \bar{b}$ ) channel. This ratio would be simultaneously sensitive to mixing and loop effects, but it is also harder to measure since it relies on vector boson fusion and suffers from low statistics. Note that an early analysis of coupling ratios was performed in [44], but it was limited by the amount of data used in the analysis and by choosing the $h \rightarrow W W^{*}$ the main channel to which all channels are compared. Of course, any systematics that affect the efficiency of signal identification will make the sensitivity of these ratios worse; a full analysis is required in order to properly estimate the final precision with which these coupling ratios can be measured.

\subsection{The possibility of a $125 \mathrm{GeV}$ Higgs}

The recent ATLAS search for the SM Higgs with $5 \mathrm{fb}^{-1}$ shows an almost $3 \sigma$ excess in the diphoton and 4-lepton channels at around $125 \mathrm{GeV}$ [19-21], and CMS has also a less significant excess in the diphoton channel at a nearby energy [22-25]. In this section we would like to contemplate what a $\sim 125 \mathrm{GeV}$ Higgs would imply for Supersymmetry and the possibility of sparticles light enough to be discovered at the LHC, should such an excess turn into a discovery.

For the MSSM, as can be seen in section 3.1, a Higgs mass at $125 \mathrm{GeV}$ can still be achieved with light stops and large enough $A$-terms. Nevertheless the parameter space becomes quite constrained, once the relatively large production cross-section that is needed to fit the current excess, as well as the bounds from $b \rightarrow s \gamma$ are taken into account. 
As can be seen comparing figure 3 and 4 , the mixing effects from $m_{A}$ have to be decoupled and it is pushed to be heavier than $800 \mathrm{GeV}$. At the same time, stops have to be heavier than at least $500 \mathrm{GeV}$ making the Higgs look very SM-like - the possibility of a natural SUSY spectrum in this case becomes more remote.

The situation changes drastically once we consider simple extensions of the MSSM where the Higgs gets new tree level mass contributions, such as the DMSSM and the NMSSM. In both these cases, large $A$-terms and heavy stops are no longer necessary to raise the Higgs mass at $125 \mathrm{GeV}$. Stops can be easily within the reach of the $7 \mathrm{TeV}$ LHC and enhance $g g \rightarrow h \rightarrow \gamma \gamma$ by $50 \%$. This enhancement is compatible with the current excess in [19-25].

\section{Conclusions}

The scenarios presented above provide a qualitative picture of the light SUSY Higgs production and decay at the LHC. The discovery of a Higgs with enhanced $\sigma \times B R$ implies that the stops are light and the $A$-terms are small by interfering constructively with the top in gluon-gluon fusion. In particular for GMSB/AMSB scenarios, current LHC and $b \rightarrow s \gamma$ bounds in combination with naturalness already favor this possibility. The mixing of $H_{d}$ with a singlet in the NMSSM also leads to an enhancement of $\sigma \times B R$ for a light mass Higgs, because it suppresses $h \rightarrow b \bar{b}$.

Conversely, the discovery of a Higgs with reduced $\sigma \times B R$ compared to its SM value may suggest that the $A$-terms are large, since stops now interfere destructively with the top in $g g \rightarrow h . B R\left(h \rightarrow \gamma \gamma\left(\right.\right.$ or $\left.\left.W W^{*}\right)\right)$ is also suppressed for a light Higgs when mixing between $H_{u}$ and $H_{d}$ increases the Higgs width to $b \bar{b}$. Finally, when a singlet is added to the Higgs sector and $\tan \beta$ is small, $H_{u}$ mixing with the singlet decreases the coupling to the top and gluon-gluon fusion is suppressed.

The size of the above effects depends on the ratio of scales between the new physics and the Higgs. The Higgs appears SM-like when the new states are pushed a few times above the Higgs mass. If a non-SM Higgs is detected at the LHC, measuring its mass and how much its couplings deviate from the SM could point to the scale of new light degrees of freedom. So, it is important to further study whether taking the ratios of different channels can improve the accuracy of Higgs coupling measurements at the LHC.

Finally, if the $\sim 125 \mathrm{GeV}$ excess observed by ATLAS persists, it can be accommodated in the MSSM with large A-terms but, when $b \rightarrow s \gamma$ bounds are taken into account, stops are pushed to be above $500 \mathrm{GeV}$ and the Higgs appears SM-like. In the MSSM, the production of a $125 \mathrm{GeV}$ Higgs is always suppressed compared to its SM value. Simple extensions of the MSSM allow for lighter stops that can now enhance the Higgs production cross-section. This enhancement may be favored by data and points to the presence of light stops in the SUSY spectrum. In this case, a $125 \mathrm{GeV}$ Higgs could be our first evidence of naturalness in SUSY. 


\section{Acknowledgments}

We would like to thank Savas Dimopoulos, Diego Guadagnoli, and Michele Papucci for useful discussions. This work was partially supported by ERC grant BSMOXFORD no. 228169 .

Open Access. This article is distributed under the terms of the Creative Commons Attribution License which permits any use, distribution and reproduction in any medium, provided the original author(s) and source are credited.

\section{References}

[1] S. Dimopoulos and H. Georgi, Softly broken supersymmetry and SU(5), Nucl. Phys. B 193 (1981) 150 [INSPIRE].

[2] ATLAS collaboration, G. Aad et al., Search for squarks and gluinos using final states with jets and missing transverse momentum with the ATLAS detector in $\sqrt{s}=7 \mathrm{TeV}$ proton-proton collisions, arXiv:1109.6572 [INSPIRE].

[3] CMS collaboration, S. Chatrchyan et al., Search for supersymmetry at the LHC in events with jets and missing transverse energy, Phys. Rev. Lett. 107 (2011) 221804 [arXiv: 1109.2352] [INSPIRE].

[4] S. Dimopoulos and G. Giudice, Naturalness constraints in supersymmetric theories with nonuniversal soft terms, Phys. Lett. B 357 (1995) 573 [hep-ph/9507282] [INSPIRE].

[5] Y. Kats and D. Shih, Light stop NLSPs at the Tevatron and LHC, JHEP 08 (2011) 049 [arXiv:1106.0030] [INSPIRE].

[6] Y. Kats, P. Meade, M. Reece and D. Shih, The status of GMSB after $1 \mathrm{fb}^{-1}$ at the LHC, arXiv:1110.6444 [INSPIRE].

[7] M. Papucci, J.T. Ruderman and A. Weiler, Natural SUSY endures, arXiv:1110.6926 [INSPIRE].

[8] Particle Data Group collaboration, K. Nakamura et al., Review of particle physics, J. Phys. G 37 (2010) 075021 [INSPIRE].

[9] R. Barbieri and G. Giudice, Upper bounds on supersymmetric particle masses, Nucl. Phys. B 306 (1988) 63 [INSPIRE].

[10] J. Fan, M. Reece and J.T. Ruderman, Stealth supersymmetry, JHEP 11 (2011) 012 [arXiv:1105.5135] [INSPIRE].

[11] J. Gunion and H.E. Haber, Higgs bosons in supersymmetric models. 1, Nucl. Phys. B 272 (1986) 1 [Erratum ibid. B 402 (1993) 567] [INSPIRE].

[12] J. Gunion and H.E. Haber, Higgs bosons in supersymmetric models. 2. Implications for phenomenology, Nucl. Phys. B 278 (1986) 449 [inSPIRE].

[13] B. Kileng, Effects of scalar mixing in gg $\rightarrow$ Higgs $\rightarrow \gamma \gamma$, Z. Phys. C 63 (1994) 87 [hep-ph/9303293] [INSPIRE].

[14] M. Spira, A. Djouadi, D. Graudenz and P. Zerwas, Higgs boson production at the LHC, Nucl. Phys. B 453 (1995) 17 [hep-ph/9504378] [INSPIRE]. 
[15] A. Djouadi, Squark effects on Higgs boson production and decay at the LHC, Phys. Lett. B 435 (1998) 101 [hep-ph/9806315] [INSPIRE].

[16] M.A. Shifman, A. Vainshtein, M. Voloshin and V.I. Zakharov, Low-energy theorems for Higgs boson couplings to photons, Sov. J. Nucl. Phys. 30 (1979) 711 [Yad. Fiz. 30 (1979) 1368] [INSPIRE].

[17] I. Low, R. Rattazzi and A. Vichi, Theoretical constraints on the Higgs effective couplings, JHEP 04 (2010) 126 [arXiv:0907.5413] [INSPIRE].

[18] M. Perelstein and C. Spethmann, A collider signature of the supersymmetric golden region, JHEP 04 (2007) 070 [hep-ph/0702038] [INSPIRE].

[19] ATLAS collaboration, Search for the Standard Model Higgs boson in the diphoton decay channel with $4.9 \mathrm{fb}^{-1}$ of ATLAS data at $\sqrt{s}=7 \mathrm{TeV}$, ATLAS-CONF-2011-161, CERN, Geneva Switzerland (2011).

[20] ATLAS collaboration, Search for the Standard Model Higgs boson in the decay channel $H \rightarrow Z Z^{(*)} \rightarrow 4 \ell$ with $4.8 \mathrm{fb}^{-1}$ of pp collisions at $\sqrt{s}=7 \mathrm{TeV}$, ATLAS-CONF-2011-162, CERN, Geneva Switzerland (2011).

[21] ATLAS collaboration, Combination of Higgs boson searches with up to $4.9 \mathrm{fb}^{-1}$ of $p p$ collisions data taken at a center-of-mass energy of $7 \mathrm{TeV}$ with the ATLAS experiment at the LHC, ATLAS-CONF-2011-163, CERN, Geneva Switzerland (2011).

[22] CMS collaboration, Combination of SM Higgs searches, PAS-HIG-11-032, CERN, Geneva Switzerland (2011).

[23] CMS collaboration, Search for the Higgs boson in the fully leptonic $W^{+} W^{-}$final state, PAS-HIG-11-024, CERN, Geneva Switzerland (2011).

[24] CMS collaboration, Search for a Higgs boson produced in the decay channel $4 \ell$, PAS-HIG-11-025, CERN, Geneva Switzerland (2011).

[25] CMS collaboration, Search for a Higgs boson decaying into two photons in the CMS detector, PAS-HIG-11-030, CERN, Geneva Switzerland (2011).

[26] P. Batra, A. Delgado, D.E. Kaplan and T.M. Tait, The Higgs mass bound in gauge extensions of the minimal supersymmetric Standard Model, JHEP 02 (2004) 043 [hep-ph/0309149] [INSPIRE].

[27] U. Ellwanger, C. Hugonie and A.M. Teixeira, The next-to-minimal supersymmetric Standard Model, Phys. Rept. 496 (2010) 1 [arXiv:0910.1785] [InSPIRE].

[28] R. Harnik, G.D. Kribs, D.T. Larson and H. Murayama, The minimal supersymmetric fat Higgs model, Phys. Rev. D 70 (2004) 015002 [hep-ph/0311349] [INSPIRE].

[29] R. Barbieri, L.J. Hall, Y. Nomura and V.S. Rychkov, Supersymmetry without a light Higgs boson, Phys. Rev. D 75 (2007) 035007 [hep-ph/0607332] [INSPIRE].

[30] CMS collaboration, Search for neutral Higgs bosons decaying to $\tau$ pairs in pp collisions at $\sqrt{s}=7 \mathrm{TeV}$, PAS-HIG-11-029, CERN, Geneva Switzerland (2011).

[31] R. Barbieri and G. Giudice, $b \rightarrow s \gamma$ decay and supersymmetry, Phys. Lett. B 309 (1993) 86 [hep-ph/9303270] [INSPIRE].

[32] M. Frank et al., The Higgs boson masses and mixings of the complex MSSM in the Feynman-diagrammatic approach, JHEP 02 (2007) 047 [hep-ph/0611326] [INSPIRE]. 
[33] G. Degrassi, S. Heinemeyer, W. Hollik, P. Slavich and G. Weiglein, Towards high precision predictions for the MSSM Higgs sector, Eur. Phys. J. C 28 (2003) 133 [hep-ph/0212020] [INSPIRE].

[34] S. Heinemeyer, W. Hollik and G. Weiglein, The masses of the neutral CP-even Higgs bosons in the MSSM: accurate analysis at the two loop level, Eur. Phys. J. C 9 (1999) 343 [hep-ph/9812472] [INSPIRE].

[35] S. Heinemeyer, W. Hollik and G. Weiglein, FeynHiggs: a program for the calculation of the masses of the neutral CP even Higgs bosons in the MSSM, Comput. Phys. Commun. 124 (2000) 76 [hep-ph/9812320] [INSPIRE].

[36] T. Elliott, S. King and P. White, Radiative corrections to Higgs boson masses in the next-to-minimal supersymmetric Standard Model, Phys. Rev. D 49 (1994) 2435 [hep-ph/9308309] [INSPIRE].

[37] G. Degrassi, P. Gambino and P. Slavich, SusyBSG: a fortran code for $B R\left[B \rightarrow X_{s} \gamma\right]$ in the MSSM with minimal flavor violation, Comput. Phys. Commun. 179 (2008) 759 [arXiv:0712.3265] [INSPIRE].

[38] B. Allanach et al., SUSY Les Houches accord 2, Comput. Phys. Commun. 180 (2009) 8 [arXiv:0801.0045] [INSPIRE].

[39] R. Kitano and Y. Nomura, Supersymmetry, naturalness and signatures at the LHC, Phys. Rev. D 73 (2006) 095004 [hep-ph/0602096] [INSPIRE].

[40] P. Lodone, Naturalness bounds in extensions of the MSSM without a light Higgs boson, JHEP 05 (2010) 068 [arXiv: 1004.1271] [INSPIRE].

[41] R. Franceschini and S. Gori, Solving the $\mu$ problem with a heavy Higgs boson, JHEP 05 (2011) 084 [arXiv: 1005.1070] [INSPIRE].

[42] L. Cavicchia, R. Franceschini and V.S. Rychkov, Supersymmetry without a light Higgs boson at the CERN LHC, Phys. Rev. D 77 (2008) 055006 [arXiv:0710.5750] [InSPIRE].

[43] M. Dührssen et al., Extracting Higgs boson couplings from CERN LHC data, Phys. Rev. D 70 (2004) 113009 [hep-ph/0406323] [INSPIRE].

[44] R. Lafaye, T. Plehn, M. Rauch, D. Zerwas and M. Dührssen, Measuring the Higgs sector, JHEP 08 (2009) 009 [arXiv:0904.3866] [INSPIRE]. 